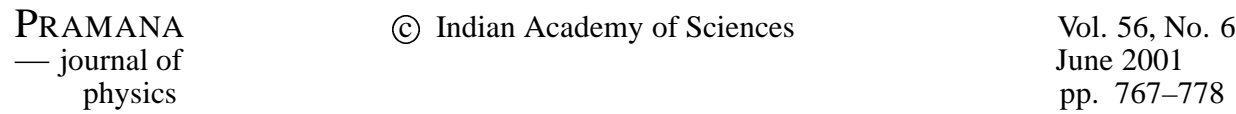

\title{
Photon transport in thin disordered slabs
}

\author{
VENKATESH GOPAL, S ANANTHA RAMAKRISHNA*, A K SOOD and N KUMAR* \\ Department of Physics, Indian Institute of Science, Bangalore 560 012, India \\ ${ }^{*}$ Raman Research Institute, C.V. Raman Avenue, Bangalore 560 080, India \\ Email: asood@physics.iisc.ernet.in; nkumar@rri.res.in
}

MS received 18 September 2000; revised 2 May 2001

\begin{abstract}
We examine using Monte Carlo simulations, photon transport in optically 'thin' slabs whose thickness $L$ is only a few times the transport mean free path $l^{*}$, with particles of different scattering anisotropies. The confined geometry causes an auto-selection of only photons with looping paths to remain within the slab. The results of the Monte Carlo simulations are borne out by our analytical treatment that incorporates directional persistence by the use of the Ornstein-Uhlenbeck process, which interpolates between the short time ballistic and long time diffusive regimes.
\end{abstract}

Keywords. Photon migration; persistent random walks; Monte Carlo simulation.

PACS Nos 42.25.Bs; 05.60.+w; 42.68.Ay

\section{Introduction}

The multiple scattering of light as it travels through a random medium is a problem that is interesting both because of the fundamental physics that it involves as well as the potential that it offers to craft novel imaging techniques for use in optically turbid media [1]. In the limit of a very large number of scattering events, the transport of the light intensity through the medium can be described by a diffusion equation which can be shown to arise out of the equation of radiative transfer [2]. In the opposite limit of only a few scattering events, single scattering theory with multiple scattering corrections is adequate [2]. It is in the intermediate regime when the transport is neither entirely diffusive nor largely ballistic that both these pictures prove insufficient. In a large scattering medium, photons scatter many times and after a few scattering events they are directionally randomized resulting in the photon performing a random walk. The transport mean free path $l^{*}$ is the length scale over which directional memory is lost. Typically, the assumption of diffusive intensity transport is a good approximation when the thickness of the medium $(L)$ is greater than about eight times $l^{*}[3,4]$, the step size of the photon random walk. The reason for the breakdown of the diffusion theory at shorter length scales can be traced to the directional persistence of the random photon walks. However, it is of importance to study alternatives to the zero-persistence diffusion theory for use in scattering media whose thickness is only a few times $l^{*}[5-7]$. 
Recently, in experiments that studied the propagation of an ultrashort pulse of light through an optically 'thin', strongly scattering slab [8], the photon diffusion coefficient $D$ measured from the temporal behaviour of the transmitted light was found to decrease as a function of the slab thickness. This result is intriguing because the diffusion coefficient should be independent of geometry. We suspect that it is the persistence of the random walks of the photons that could cause this apparent reduction of the diffusion coefficient and we explore this possibility. Monte Carlo simulations of the random walks naturally account for the persistence arising due to constant speed [6] and finite mean free paths of the photons. Analytically, we have attempted to incorporate this persistence by the use of the Ornstein-Uhlenbeck (OU) process of Brownian motion.

We should note here that we are dealing with the case of light (which is a wave) propagating in a random medium, where the randomness may be in space (quenched disorder), but treat it as the Brownian motion of a classical particle i.e., temporal disorder or a stochastic process. This approximation of an incoherent energy transport is valid in the weak scattering limit $\left(k l^{*} \gg 1\right.$, where $\left.k=2 \pi / \lambda\right)$.

In this paper, we describe the results of our Monte Carlo simulations to study photon transport in thin slabs. We show that in small slabs, photons that traverse large paths are forced by the constrained geometry to travel in paths that loop back upon themselves, thus lowering the rate at which the photons are transported in the medium. In our effort to find alternatives to the widely used zero-persistence diffusion picture of transport, we examined the Ornstein-Uhlenbeck theory of Brownian motion. By making an ansatz, we found the OU process to yield close agreement with the results of our simulations. We find the OU process to adequately describe the ballistic motion at short times and become identical with the diffusion approximation asymptotically at long times.

The paper is organized as follows. In $\S 2$, the Monte Carlo simulation technique used is described. Our results, qualitatively explaining the reduced photon diffusion coefficient in confined geometries are presented in $\S 3$. In $\S 4$ we describe the Ornstein-Uhlenbeck process and the modifications made to use it to describe the transport of multiply scattered light. We also compare results obtained using the OU process with those from randomwalk simulations of photon transport. Finally, we conclude in $\S 5$.

\section{Monte Carlo simulations}

The procedure for our Monte Carlo simulations was as follows. Photons were launched from the centre of a slab in the $+z$ direction. The slab was of infinite transverse extent but with a finite thickness $L$ in the $z$ direction. We chose the centre of the box as the source of the photons as the results are then easier to interpret. The simulation modelled photon transport assuming that the photons travel exponentially distributed lengths between scattering events. The probability $P(s)$ of travelling a ballistic path length $s$ is given by the familiar Lambert-Beer law, $P(s)=\exp \left(-s / l_{\mathrm{s}}\right)$, where $l_{\mathrm{s}}$ is the scattering mean free path of the photons in the medium. The scattering mean free path is set by the scattering cross-section $\sigma$ and the number density $\phi$ of the scatterers as $l_{\mathrm{s}}=1 / \sigma \phi$. The scattering cross-sections were calculated using Mie theory [9]. The random paths between scattering events were generated taking $s=-l_{\mathrm{s}} \cdot \ln (\mathcal{R})$, where $\mathcal{R}$ is a random number uniformly distributed between 0 and 1 [10]. The scattering angles were chosen such that they had a distribution of directions given by the Henyey-Greenstein phase function, where the prob- 
ability of scattering at an angle $\theta$ relative to the incident direction of the photon is given by

$$
P(\cos \theta)=\frac{1-g^{2}}{\left(1+g^{2}-2 g \cos \theta\right)^{3 / 2}},
$$

and where $g=\langle\cos \theta\rangle=1-\left(l_{\mathrm{s}} / l^{*}\right)$ is the scattering anisotropy. Thus, after being launched from the centre of the slab, photons were repeatedly scattered until they encountered a boundary, at which point they were assumed to have escaped from the slab and a new photon was launched from the centre. Each such random walk trajectory generated was stored. Time dependent statistics were calculated by dividing these trajectories into numerous slices of equal lengths and hence equal intervals of time. Simulations were performed for three different scattering anisotropies $(g=0.06,0.423$ and 0.732$)$ to study the range from nearly isotropic to highly anisotropic scatterers.

As a check on the accuracy of our simulations, the diffuse transmission probability [11] as a function of the slab thickness for slabs thicker than $5 l^{*}$ and the photon diffusion coefficient $D_{0}=c l^{*} / 3$ were calculated. Excellent agreement with theoretical predictions was obtained in both cases.

\section{Photon diffusion in confined geometries}

We begin by providing a qualitative description of the motion of the photon cloud at short times, which we then support with data from our simulations. Consider a source of photons at the centre of a slab of scattering material and being launched in the $+\mathrm{z}$ direction. A characteristic time scale in the problem is the time $t_{b}=L / c$, that it would take an unscattered or ballistic photon to traverse the thickness of the slab. At very short times, $\left(t<t_{b}\right)$, there are two kinds of photons, those that are ballistic and have not yet been scattered and those that have. There is a large separation in the rate at which these photons are moving away from the source. At long enough times, gradually, all photons suffer scattering events and a smooth distribution of distances from the source is obtained, but at short times there is a sharp distinction between ballistic and multiply scattered photons.

If now the scattering takes place in a confined geometry, before a smooth distribution of radial distances from the origin is allowed to form, then a photon that must multiply scatter and yet stay within the confines of the scattering medium must necessarily follow a tortuous path that loops around and 'doubles back' on itself. As we show later, this leads to a reduction of our diffusion coefficient, for if the photon does not 'loop back', it exits the slab along with or just after the ballistic pulse. For these paths that loop back upon themselves, the rate at which the radial distance from the origin, a 'front velocity' of the photon cloud as it were, is clearly increasing at a much slower rate than it would in an unconfined medium. On increasing the size of the slab though, the number of ballistic photons decreases exponentially and the well known smooth path length distribution from the origin for diffusion is obtained [3]. Figures 1 and 2, which we now describe, support this picture.

In the random walk simulations, we probed the rate of transport of photons in the medium by investigating the mean square displacement of the photons as a function of time. This is reflected in the rate at which the photon cloud in the medium expands, the 

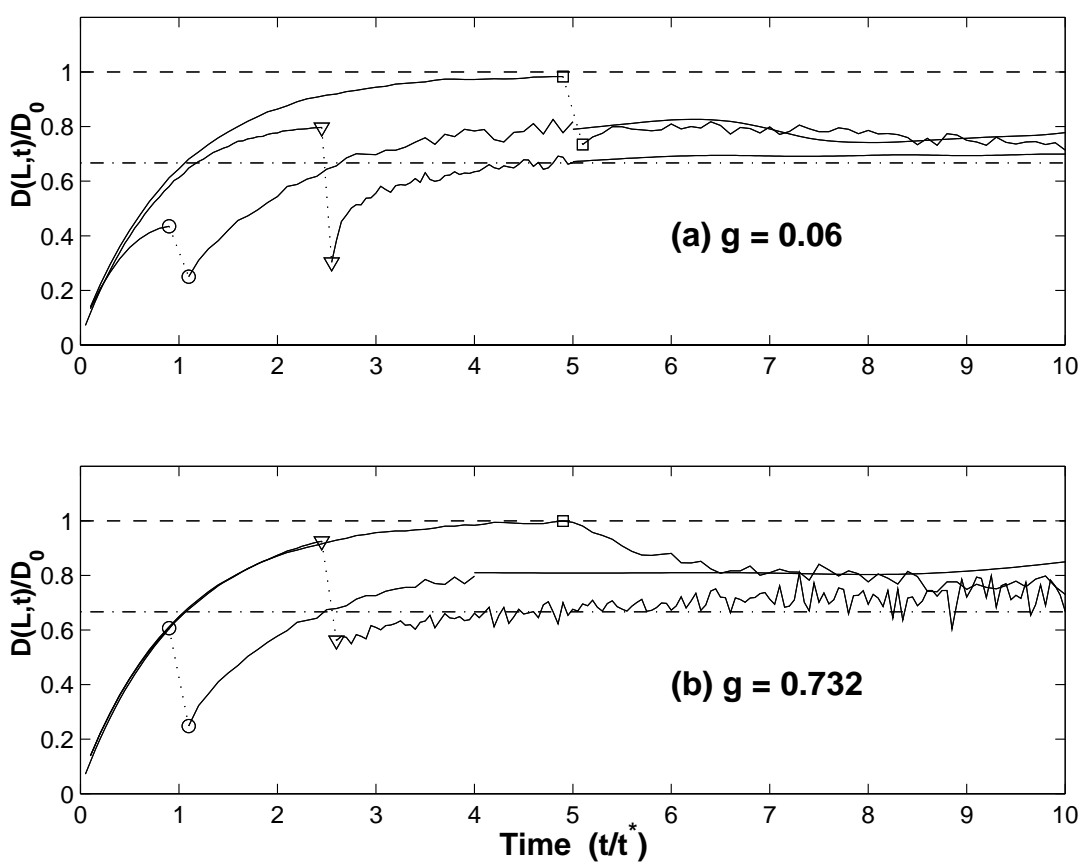

Figure 1. Variation of the normalized local slope of the mean square displacement $D(t ; L)=\frac{1}{6 D_{0}} \frac{\mathrm{d}\left\langle\Delta r^{2}\right\rangle}{\mathrm{d} t}$ with time for different values of the optical density $L / l^{*}$. The $\mathrm{x}$-axis is in units of the randomization time $t^{*}=l^{*} / c$. In the thinnest slab, very few photons survive at long times and thus the statistics are very poor. This results in large fluctuations in the local slope of the mean square displacement. To avoid cluttering the rest of the figure we have fitted a smoothing spline curve to the noisy data. This brings out the fact that at long times the photons are effectively performing a random walk in two dimensions and $D(t ; L)$ converges to 0.66 (denoted by the dash-dotted line). The symbols correspond to the following slab thicknesses: $\circ-2 l^{*}, \nabla-5 l^{*}, \square-10 l^{*}$.

mean square displacement simply being the mean radius of the photon cloud. Photons were propagated in slabs of varying thicknesses as described previously. Some of the results are shown in figure 1 . In both figures $1 \mathrm{a}$ and $1 \mathrm{~b}$, we show the local slope of the mean square displacement denoted by an effective diffusion constant $D(t ; L)$ normalized to $6 D_{0}$, as a function of time. At long times, and for large slabs, we have the well-known Einstein result, $\left\langle\Delta r^{2}\right\rangle=6 D_{0} t$, where $D_{0}=c l^{*} / 3$ is the photon diffusion coefficient in an infinite medium, and the local slope thus tends to unity. Figure 1a shows the variation of this local slope for nearly isotropic scatterers with a scattering anisotropy $g=0.06$ for different slab thicknesses, while figure $1 \mathrm{~b}$ shows the same data for anisotropic scatterers with $g=0.732$. All the curves show a sharp break marked by a symbol. This is the point at which the ballistic photons escape from the slab. This results in a sharp decrease in the mean square displacement leading to an artificial negative value for the diffusion coefficient, which is avoided by showing the slopes just before and after the escape of the ballistic pulse. 

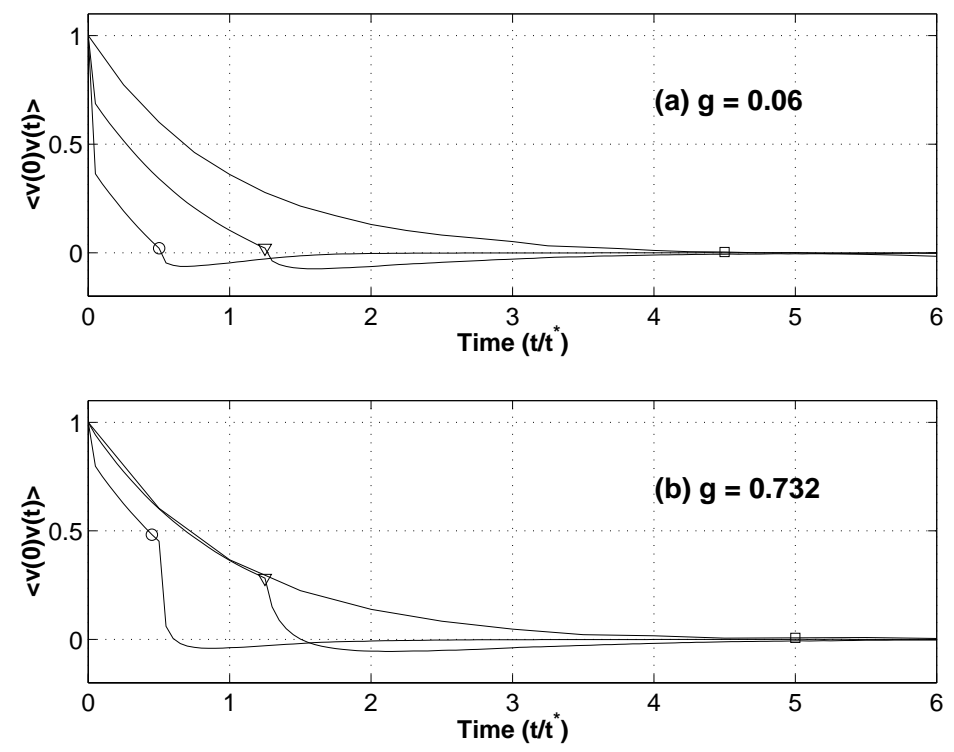

Figure 2. Normalized velocity autocorrelation function as a function of normalized time $t^{*}$, for two values of the scattering anisotropy.

In both figures $1 \mathrm{a}$ and $1 \mathrm{~b}$, the circles denote slabs of thickness $2 l^{*}$, the triangles slabs of $5 l^{*}$ and the squares $10 l^{*}$.

The main features of figure 1 are that at short times, photon motion is ballistic and the mean square displacement increases quadratically in time (i.e., $D(t ; L) \propto t$ ). At long times, for large slabs, the motion is diffusive and the local slope is unity. After most photons have escaped from the slab, the only surviving photons in the medium are those that are confined to the transverse plane and the local slope is that corresponding to two dimensional diffusion, i.e. $D\left(t \gg t_{b}\right)=2 D_{0} / 3$. At long times it can be seen that the normalized local slope tends to $2 / 3$.

To show that the multiply scattered photons in the medium undergo long circular scattering loops, we investigated the normalized velocity autocorrelation function (VACF) for photons in the medium, which are shown in figure $2 \mathrm{a}(g=0.06)$ and figure $2 \mathrm{~b}(g=0.732)$. The calculation of the VACF $C(t)=\langle\mathbf{v}(0) \cdot \mathbf{v}(t)\rangle /\langle\mathbf{v}(0) \cdot \mathbf{v}(0)\rangle$ is performed as follows. Each photon trajectory is divided into a number of sections corresponding to the resolution in time that is chosen. At the origin of each such 'time step', the angle made by the path at that point with the global coordinate system, which is equivalent to the angle made by the velocity vector, is stored. The correlation function $C(t)$ is now formed and averaged over all random walk trajectories. Once again the symbols denote the points at which the ballistic pulse exits the slab and the circles, triangles and squares represent the same slab thicknesses as in figure 1. As soon as the ballistic pulse leaves the medium we see that the VACF is negative, indicating that the photons have on average, reversed their direction of motion, exactly what would be expected for photons travelling such 'loop-like' paths as we have stated. That the average is influenced shows that we are observing a feature common 
to a majority of the photons and not just the reversal of a few trajectories. This is to be contrasted with the VACF observed for large slabs where the function is always positive and smoothly decays to zero.

In figure 3 , we plot the value of the normalized local slope $D(t ; L)$ just after the escape of the ballistic pulse. We believe that since this quantity is indicative of the rate at which photon transport occurs in the medium, it must be reflected in a pulse transmission experiment in the rate at which photons are transported across the boundary of the slab. The resulting figure is qualitatively similiar to the data presented in [8]. It should however, be noted that we derive the diffusion coefficient $D$ from the behaviour occurring over relatively short times $\left(<10 t^{*}\right)$, whereas, in [8], the decay of the transmitted pulse occurs over longer times (up to $100 t^{*}$ ). Thus, it appears as if the two phenomena are different, occurring over different time regimes. The effect of the short time behaviour on the overall fitting of the data in [8], however, cannot be ignored.

While it is well understood that accounting for reflections at the sample boundary is of great importance in the application of the diffusion approximation [12-15], it must be pointed out that to keep details to a minimum, we have not accounted for interfacial reflections in our simulations. The effect of a boundary reflectivity would be to re-inject a small part of the escaping pulse back into the medium and thus create a small number of photons that would have even larger residence times.

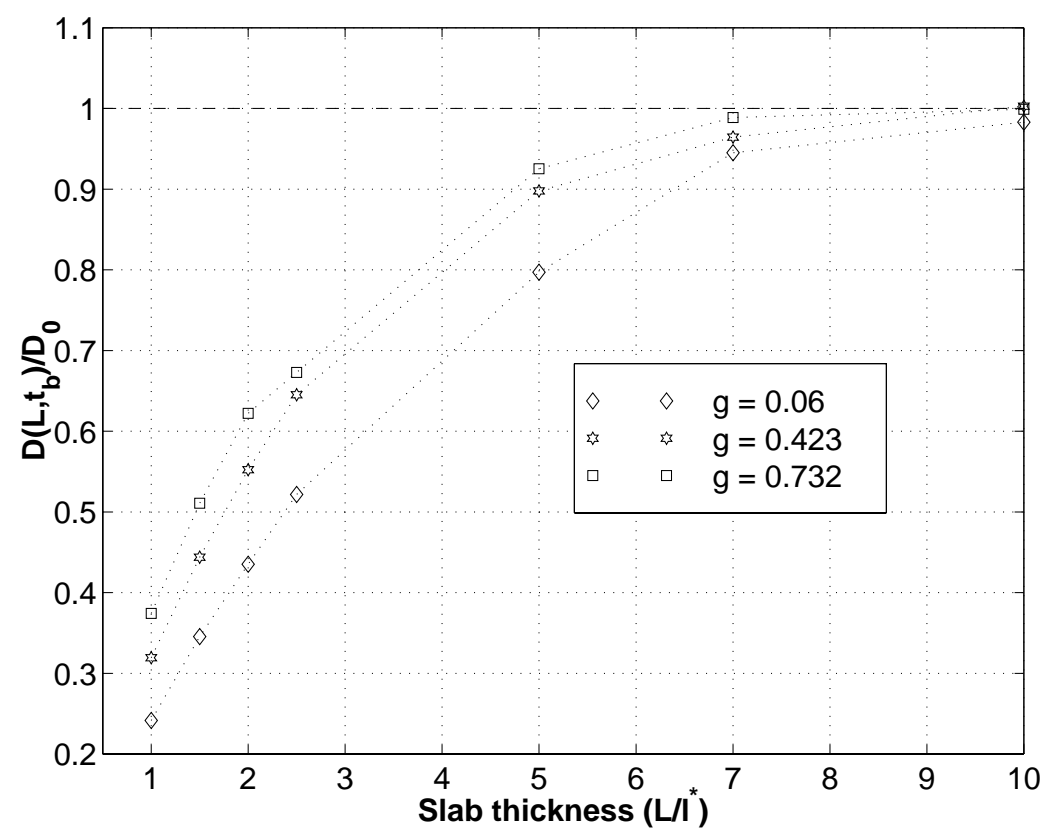

Figure 3. The value of the normalized local slope $D(t ; L)$ just prior to the escape of the ballistic pulse is taken to approximate the rate of diffusion in the medium and is plotted as the effective diffusion constant for slabs of varying thicknesses. The slab thicknesses have been scaled by the transport mean free path $l^{*}$. 


\section{The Ornstein-Uhlenbeck process}

The Ornstein-Uhlenbeck process describes the stochastic behaviour of the velocity of a Brownian particle. The motion of the particle is described by the stochastic Langevin equation $\ddot{\vec{X}}+\beta \dot{\vec{X}}=\vec{A}(t)[16]$. The friction coefficient $\beta$ and the components of the concomitant Gaussian white noise $\vec{A}(t)$ are related as $\left\langle A_{i}(t) A_{j}(t+\tau)\right\rangle=\left(2 \beta k_{\mathrm{B}} T / m\right) \delta(\tau) \delta_{i j}$ so as to be consistent with the condition of thermal equilibrium with the thermal bath at temperature $T$, where $k_{\mathrm{B}}$ is the Boltzmann constant and $m$ is the mass of the particle. The quantity relevant to our purposes is the probability density $P_{\infty}\left(\vec{r}, t ; \overrightarrow{r_{0}}, \overrightarrow{u_{0}}, t=0\right)$ such that $P_{\infty}\left(\vec{r}, t ; \overrightarrow{r_{0}}, \overrightarrow{u_{0}}, t=0\right) d^{3} r$ is the probability of finding the particle in the volume element $d^{3} r$ at a position $r$ at time $t$; given that the particle was at $\overrightarrow{r_{0}}$ with a velocity $\overrightarrow{u_{0}}$ at a time $t_{0}(=0)<t$. The $P_{\infty}\left(\vec{r}, t ; \overrightarrow{r_{0}}, \overrightarrow{u_{0}}, t=0\right)$ for an infinite medium is given by [16]

$$
\begin{aligned}
P\left(\vec{r}, t ; \overrightarrow{r_{0}}, \overrightarrow{u_{0}}, t=0\right)= & {\left[\frac{m \beta^{2}}{2 \pi k_{\mathrm{B}} T f(t)}\right]^{3 / 2} } \\
& \times \exp \left[-\frac{m \beta^{2}}{2 k_{\mathrm{B}} T f(t)}\left|\vec{r}-\overrightarrow{r_{0}}-\frac{\overrightarrow{u_{0}}}{\beta}\left(1-\mathrm{e}^{-\beta t}\right)\right|^{2}\right],
\end{aligned}
$$

where $f(t)=2 \beta t-3+4 \mathrm{e}^{-\beta t}-\mathrm{e}^{-2 \beta t}$.

The basic motivation to adapt this process to light transport is the following. The diffusion approximation (Wiener process) is valid in the limit when $l^{*} \rightarrow 0$ and $c \rightarrow \infty$ such that the diffusion coefficient $D_{0}=c l^{*} / 3$ is a constant. Multiply scattered photons on the other hand have a finite mean free path in the medium and are scattered only after intervals of ballistic flight. Some alternatives including a generalized Telegrapher equation [5] have been proposed to account for the transition from ballistic motion to diffusive transport. It should be noted that such a generalization to higher dimensions (i.e. merely by replacing $\partial^{2} / \partial x^{2}$ with $\nabla^{2}$ ), however, is not quite correct, and certainly not correct for short times when ballistic propagation dominates over diffusion. Indeed, it has also been shown that such a generalized Telegrapher equation provides no better an approximation than the diffusion theory at short time and length scales in higher dimensions by comparing with Monte Carlo simulations [17]. The OU process accounts for a finite speed of propagation by making a more physical assumption for Brownian particles, that of assuming a speed distribution for the particles with a well defined mean speed, thus avoiding the unphysical infinite velocity built into the diffusion equation and therefore interpolating between the short time ballistic motion $\left(\left\langle\Delta r^{2}\right\rangle \sim t^{2}\right)$ and the long time diffusive limit $\left(\left\langle\Delta r^{2}\right\rangle \sim t\right)$. Also the particle retains directional memory for a time $\sim t^{*}\left(=\frac{c}{l^{*}}\right)$ and the effects of a finite $l^{*}$ are thus accounted for. This makes it attractive to consider the OU process to obtain a transport equation that would describe transport with a finite mean free path and finite speed, and hence capable of describing both the ballistic and diffuse components.

Hence we force the following identifications as an ansatz and equate the root mean square (r.m.s.) speed and the initial speed $u_{0}$ of the photons, to $c$, the speed of light in the medium. We recognize, of course, that there is no thermal bath nor inertia for light, and that equating the r.m.s. speed of the photons to the speed of light in the medium is a formal identification forced on us by the OU stochastic process introduced here to approximate the diffusion of light. Thus our ansatz becomes $\left\langle\vec{u}^{2}\right\rangle=c^{2}=\frac{3 k_{\mathrm{B}} T}{m}, D=\frac{c l^{*}}{3}=\frac{k_{\mathrm{B}} T}{m \beta}$, and $\frac{\left|\overrightarrow{u_{0}}\right|}{\beta}=\frac{c}{\beta}=l^{*}$. Rewriting the probability distribution (2) using these we get 


$$
\begin{aligned}
P_{\infty}\left(\vec{r}, t ; \overrightarrow{r_{0}}, \overrightarrow{u_{0}}, t=0\right)= & {\left[\frac{3}{2 \pi l^{* 2} f(t)}\right]^{3 / 2} } \\
& \exp \left[-\frac{3}{2 l^{* 2} f(t)}\left|\vec{r}-\overrightarrow{r_{0}}-l^{*} \hat{n}\left(1-\mathrm{e}^{-c t / l^{*}}\right)\right|^{2}\right] .
\end{aligned}
$$

It should be noted that in modelling the random walk of the photons by the OU process, we have implicitly allowed the speed of the photon to fluctuate as it propagates through the medium. It should also be remarked here that some of the attempts made to enforce the strong constraint of constant photon speed have had only partial success, in that they could calculate the probability distribution subject to the speed constraint only in the weaker (average) sense i.e., $\int_{0}^{t}\left[\left(\frac{\mathrm{d} \vec{r}}{\mathrm{~d} t}\right)^{2}-c^{2}\right] \mathrm{d} t=0$ [18]. A path integral approach shows that the finite r.m.s speed defined by the fluctuation-dissipation theorem for the OU process is a stronger global constraint than the speed constraint imposed in [18]. An exact theory for the photon diffusion-at-a-constant-speed with a locally fixed speed was recently formulated by us [6], where the deficiencies of the zero-persistence diffusion theory were overcome. But we could obtain only approximate analytical solutions and exact numerical solutions. In this context, the simplicity of the analytical solutions to the OU process which also incorporates some persistence in the velocity space makes it important.

The probability distribution function for a finite slab with absorbing boundary conditions at $z=0$ and $z=L$ is approximately expressed in terms of $P_{\infty}$ by the method of images $[19,20]$ yielding

$$
\begin{aligned}
P_{L}\left(\vec{r}, t ; \overrightarrow{r_{0}}, \overrightarrow{u_{0}}, t=0\right)= & \sum_{n=-\infty}^{+\infty}\left[P_{\infty}\left(\vec{r}+2 n L \hat{z}, t ; z_{0} \hat{z}, c \hat{z}, t=0\right)\right. \\
& \left.-P_{\infty}\left(\vec{r}+2 n L \hat{z}, t ;-z_{0} \hat{z},-c \hat{z}, t=0\right)\right] .
\end{aligned}
$$

This solution for absorbing boundaries holds only approximately in the limits which we explain below. The equation for the marginal probability distribution for the position does not remain invariant when the initial velocity $\overrightarrow{u_{0}}$ and the initial position $\overrightarrow{r_{0}}$ are changed. More physically, for diffusive motion, all paths have an equal probability of occurrence and it is for this reason that the method of images can be applied. In the OU process on the other hand, paths retain for a time $t^{*}$, due to inertia, a 'memory' of their initial direction. Thus at times short compared to the randomization time $t^{*}$ or when the distance between the source and the absorbing boundary is less than the transport mean free path, the method of images is not strictly valid as there is an imperfect cancellation of forbidden photon paths and their mirror images. This error however decreases exponentially with increasing slab thickness and with increasing time. Also, as can be seen in our results, the errors are small enough to be neglected for the thicknesses we have considered $\left(L \geq 2 l^{*}\right)$ with the source located at the centre of the slab. A rigorous solution but for a semi-infinite half space only with a single absorbing boundary is given in [21]. The series (4) is absolutely and uniformly convergent. The normalization $\int P_{L}\left(\vec{r}, t ; \overrightarrow{r_{0}}, \overrightarrow{u_{0}}, t=0\right) \mathrm{d}^{3} r$ reduces with time, corresponding to the flux of probability density which leaks out of the slab.

In the diffusion approximation, one imposes the absorbing boundary conditions not at the physical boundaries but at extrapolated boundaries at a distance $z_{\mathrm{e}}=2 l^{*} / 3$ outside the slab [22,19]. At times long compared to the 'randomization time' $\left(t>t^{*}\right)$, the solution should match with the diffusion approximation. However, for short times $\left(t \sim t^{*}\right)$, 

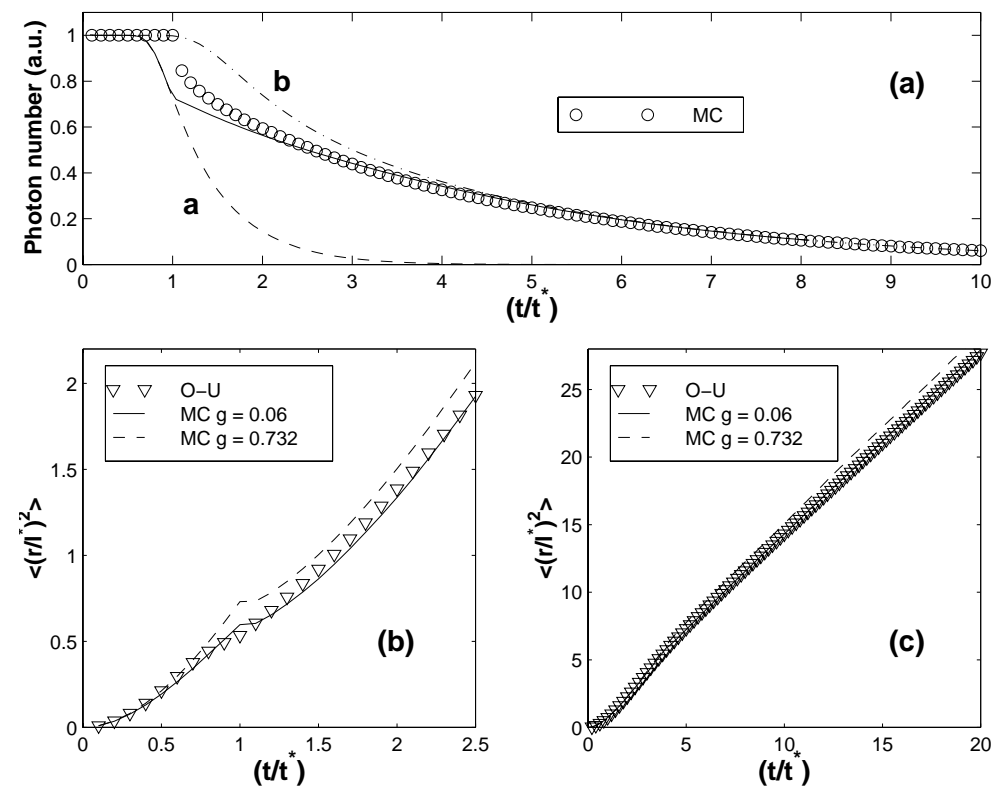

Figure 4. Comparison of results obtained by modelling photon transport using the Ornstein-Uhlenbeck process with those obtained from random walk simulations. Figure 4a compares the rate at which photons exit the slab when different boundary conditions are applied. The lower figures compare the mean square displacement calculated by integrating equation (4) with the random walk simulations for different slab thicknesses. Figure $4 \mathrm{~b}$ shows a thin slab where the diffusion approximation is not valid while figure $4 \mathrm{c}$ is for a slab where the transport is mainly diffusive.

the photons are ballistic and traverse only the true thickness of the medium. In the absence of a comprehensive theory for the boundary position, we adopt the following interpolation scheme. The extrapolated boundary is kept at the physical boundary at short times up to $t=t^{*}$ after which it is smoothly moved to $z_{\mathrm{e}}$ outside the physical boundary asymptotically as $t \rightarrow \infty$, giving an effective slab thickness of

$$
L_{\text {eff }}=L+2 \theta\left(t-t^{*}\right)\left[1-\exp \left\{-\left(t / t^{*}-1\right)\right\}\right] z_{\mathrm{e}},
$$

where $\theta$ is the Heaviside step function. It should be noted that a fitting parameter of the order of unity could have been used to determine the time at which the boundary starts to move. However, only a qualitative understanding is being attempted and such a parameter is unnecessary.

Figure 4 encapsulates the effectiveness of the use of the OU process. Figure $4 \mathrm{a}$ shows graphically the effect of the various boundary conditions. The circles represent the number of photons in the slab as a function of time, normalized to 1, obtained from Monte Carlo simulations for a slab thickness of $L=2 l^{*}$ for nearly isotropic scatterers with $g=0.06$. The curve marked 'a' shows the result when the extrapolated boundaries are maintained 
at the physical boundaries of the cell. As can be seen, while this curve approximately captures the time at which photons begin to escape from the cell and the photon number density begins to reduce, it completely fails to fit the long time diffuse tail. The curve marked ' $b$ ', is one in which the extrapolated boundaries are held at the extrapolation length $z_{\mathrm{e}}=2 l^{*} / 3$ throughout. Here, there is excellent agreement at long times with the Monte Carlo data but the pulse exits the medium much later than the ballistic pulse, a consequence of the ballistic pulse having to traverse a medium whose thickness is $L+2 z_{\mathrm{e}}$. The solid line is the result of our moving boundary conditions which fits curve 'a' almost exactly at short times and agrees very well with the Monte Carlo data at long times. It is to be noted though that even when the extrapolation length is set to zero, the ballistic pulse exits the slab faster than a true ballistic pulse would do. This is a consequence of the fact that we model the photons as having a distribution of speeds. As a result, there are photons that are travelling with a speed greater than the speed of light in the medium resulting in this artefact of a 'pre-ballistic' pulse. As the slab thickness is increased this effect becomes vanishingly small since there are almost no ballistic photons in the medium. However, it is important to appreciate that the OU process describes most of the essential features of the simulation at short times which would not be possible using the diffusion approximation.

Figure $4 \mathrm{~b}$ compares the results obtained for the mean square displacement of the photons from the point at which they are launched, as a function of time, for a cell of thickness $L=2 l^{*}$. At short times the transport is predominantly ballistic and the mean square displacement shows the characteristic quadratic behaviour. The kink in the curves occurs when the ballistic photons exit the slab. At this point, the fastest moving photons are lost and thus the average value of the mean square displacement is sharply lowered. The OU process compares well with the Monte Carlo data. Figure $4 \mathrm{c}$ shows the same data but for a cell whose thickness $L=8 l^{*}$. Now the regime is one where the diffusion approximation is valid and excellent agreement is obtained between the OU process and Monte Carlo data.

Thus, despite the fact that the method of images is not strictly valid for early times, we find that the OU process proves reasonably effective in capturing most of the features of photon transport in confined geometries, a fact that we feel deems it worthy of further study.

\section{Conclusions}

We have, using Monte Carlo simulations of random walking photons, investigated the effect of a confined geometry on the conversion of ballistic motion to diffusive transport in a multiply scattering medium. We find that while most photons exit the medium unscattered or scattered but nearly undeviated from their original direction, a small fraction of the photons are forced to travel along paths that close upon themselves. Due to the directional persistence of the random photon walks and the confined geometry, there is an autoselection of only photons which are effectively travelling backwards to remain within the slab. This looping motion is clearly visible in the velocity autocorrelation function $C(t)$, where one observes a negative dip in the correlation function indicating a reversal of direction. This reversal of direction retards the mean rate at which these multiply scattered photons are transported through the medium. This reduced mean rate of transport could be reflected in pulse transmission experiments as an apparent reduction of the photon diffusion coefficient. Thus we find a breakdown of the pure diffusion model in thin slabs and at short 
times scales $\left(\sim l^{*} / c\right)$. The reason being that the mean square displacement is, on these time scales, necessarily quadratic in time (i.e. ballistic transport), unlike the long time behaviour which is diffusive. In this work we have examined the effect of the cross-over from the short time ballistic to the long time diffusive motion on photon transport by defining an effective diffusion constant based on the ensemble of trajectories that remain within the medium (sub-ensemble average) until a time $t$.

We have also investigated the suitability of modelling photon transport by the OrnsteinUhlenbeck process. We have attempted to use the OU process in contrast to a Wiener process with an unbounded speed so as to incorporate the effects of a finite mean free path and hence, persistence in the random photon walks, while also implementing the constancy of the speed of the photon in a weak average sense. We have also proposed approximate solutions of the OU process for absorbing boundaries based on the mirror image method. This yields very good agreement with data obtained from our randomwalk simulations. In view of the simplicity and accuracy of the approximate solutions for absorbing boundaries, this should prove to be an important and useful alternative to the diffusion equation in the intermediate scattering regime when $2 l^{*} \leq L \leq 8 l^{*}$. Finally, we would like to clarify the underlying idea of our approach. In all such treatments of the problem of wave propagation in random media as a random walk problem (which is valid under certain conditions) one chooses a process which models the stochastic, kinematic aspects as closely as possible. As we have pointed out earlier, we are not implying a literal, naive acceleration/deceleration of a 'massive photon', but only that modelling it as a OU process imposes a finite mean free path and a finite speed of the photon in the problem. The OU process manages to impose the constraint of a fixed speed of the photon in the sense of a weak global constraint. Further work is in progress to extend these ideas to diffusing wave spectroscopy in optically 'thin' samples.

\section{Acknowledgements}

The authors would like to thank the Supercomputer Education and Research Centre (SERC) at the Indian Institute of Science for computational facilities. AKS thanks the Raman Research Institute for a visiting professorship. SAR would like to thank Prof. Rajaram Nityananda for very useful discussions.

\section{References}

[1] A Yodh and B Chance, Phys. Today 51, 34 (1995)

[2] Akira Ishimaru, Wave propagation and scattering in random media (Academic, New York, 1978) vols 1 and 2

[3] D J Pine, D A Weitz, J X Zhu and E Herbolzheimer, J. Phys. (Paris) 51, 2101 (1990)

[4] K M Yoo, F Liu and R R Alfano, Phys. Rev. Lett. 64, 2647 (1990)

[5] D J Durian and J Rudnick, J. Opt. Soc. Am. A14, 235 (1997)

[6] S Anantha Ramakrishna and N Kumar, Phys. Rev. E60, 1381 (1999)

[7] M Boguñá, J M Porrà and J Masoliver, Phys. Rev. E58, 6992 (1998)

[8] R H J Kop, P de Vries, R Sprik and A Lagendijk, Phys. Rev. Lett. 79, 4369 (1997)

[9] C F Bohren and D R Huffman, Absorption and scattering of light by small particles, 
Appendix-A (Wiley Interscience, New York, 1983). Also available by anonymous ftp from $\mathrm{ftp} / / /$ astro.princeton.edu/draine/scat/bhmie

[10] W H Press, S A Teukolsky, W T Vetterling and B P Flannery, Numerical recipes in FORTRAN - The art of scientific computing (Cambridge 1992)

[11] D J Durian, Phys. Rev. E51, 3350 (1995)

[12] A Lagendijk, R Vreeker and P de Vries, Phys. Lett. A136, 81 (1989)

[13] K A Kaplan, M H Kao, A Yodh and D J Pine, Appl. Opt. 32, 3828 (1993)

[14] M Ospeck and S Fraden, Phys. Rev. E49, 4578 (1994)

[15] J X Zhu, D J Pine and D A Weitz, Phys. Rev. E44, 3948 (1991)

[16] S Chandrasekhar in Selected papers on noise and stochastic processes edited by Nelson Wax (Dover, New York, 1954)

[17] J M Porrà, J Masoliver and G H Weiss, Phys. Rev. E55, 7771 (1997

[18] L T Perelman, J Wu, I Itzkan and M S Feld, Phys. Rev. Lett. 72, 1341 (1994)

[19] P M Morse and H Feshbach, Methods of theoretical physics (McGraw Hill, New York, 1953) vol. I

[20] W Feller, An introduction to probability theory and its applications (Wiley, New York, 1950) vol. I

[21] T W Burkhardt, J. Phys. A26, L1157 (1993)

[22] P A Lemieux, M U Vera and D J Durian, Phys. Rev. E57, 4498 (1998) 